\title{
A GLOBAL CROP GROWTH MONITORING SYSTEM BASED ON REMOTE SENSING
}

\author{
Meng Ji-hua, Wu Bing-fang, Li Qiangzi \\ Institute of Remote Sensing Applications, Chinese Academy of Sciences, Beijing, P.R. China- mengjh@irsa.ac.cn
}

Commission VII, SS-7

KEY WORDS: Remote Sensing, global crop growth monitoring, system

\begin{abstract}
:
Crop growth means the growth of cereal crop seedlings, as well as the status and trend of their growth. It has been one of the most important aspects of agricultural remote sensing in the last twenty years. Evaluation of crop growing condition in large scope before harvesting could be helpful for field management, as well as provide important information for early crop production estimation. For a country with the population of 1.2 billion it is important to know not only the domestic crop growth, but also the crop condition in other big agricultural countries that have food trade with China. This paper introduced the design, methods used and implementation of a global crop growth monitoring system, which satisfies the need of the global crop monitoring in the world. The system uses two methods of monitoring, which are real-time crop growth monitoring and crop growing process monitoring. Real-time crop growth monitoring could get the crop growing status for certain period by comparing the remote sensed data (NDVI, for example) of the period with the data of the period in the history (last year, mostly). The differential result was classified into several categories to reflect the condition at difference level of crop growing. This analysis result allows users to quickly assess how much and where conditions have either deteriorated, remained unchanged or improved. The crop growing process monitoring is the contrast between year and year for crop growth profile, which reflects the crop growing continuance at time during crop growing season. Time series of NDVI during the crop season are used and crop growth profiles are formed by getting statistical average of time series NDVI image for plowland in certain regions such as a state. Eigenvalues such as growing rate, peak value, average and so on were gotten from the crop growing profile to estimate crop growing status which concerns the whole growing process. Based on the above monitoring methods, we developed a crop growth monitoring system based on remote sensing, which provides a monitoring and analyzing environment for real-time crop growth monitoring and crop growing process monitoring to all the users. The system was developed in $\mathrm{C} / \mathrm{S}$ pattern and includes five main functional modules, which are real-time crop growth monitoring module, crop growing process monitoring module, results visualization module, business management module and system configuring module. The structure and the function are particularly described in the paper. In the system, both real-time crop growth monitoring and crop growing process monitoring are carried out at three scales, which are state (province) scale, country scale and continent scale. While in most of the countries in the world the monitoring was carried out at country scale, monitoring in large agricultural countries such as USA, Canada, India, etc, was carried out at both state (province) scale and country scale. This can provide more detailed crop growth information in these countries. Much more macro crop growth information was supplied by running the system at continent scale such as North America, Europe, Africa and South East Asia, etc. Taken 10-day (16-day for MODIS) composite NDVI products of NOAA/AVHRR, SPOT/VEGETATION and MODIS as data source, the system has been run successfully for more than a year, which provides decision-supporting information on crop condition to more than a dozen of ministries and commissions in China.
\end{abstract}

\section{INTRODUCTION}

It is of great significance to obtain the crop condition information at early stages in the crop-growing season, especially in those big agricultural countries that has trade on grain with us. Sometimes it is even more important than acquiring the exact production after harvest time. Regional crop growth estimates based on filed reports are often expensive, prone to large errors, and cannot provide real-time, spatially explicit estimates or forecasting of crop condition needed to establish food policies. Satellite systems provide temporally and spatially continuous data over much of the globe using relatively few instruments. Along with the development of remote sensing applications, satellite data has become the uppermost data source to monitor large-scale crop condition. USDA of U.S. and VI of EU, as well as FAO, all have build their own crop monitoring systems based on remote sensing (Liu Haiqi, 1999; Rassmussen, 1997). After researching in the field for more than 20 years, we have accumulated abundant experience in monitoring methods and system construction $\mathrm{(Wu}$

This research was supported by the Knowledge Innovation Programs of the Chinese Academy of Sciences (No. KSCX1-YW-0901 ) and National 863 Program Project (No. 2003AA131050)
Bingfang, 2004; Yang Bangjie, 2002). In this condition we built a global crop growth monitoring system which can monitor the crop condition in the 46 main grain-producing countries around the world. These monitoring results help a lot to relevant departments when they are making decision on international trade of food and grain.

The structure, function and the run of the system, as well as the methods used, are described in detail in this paper.

\section{MONITORING METHODS USED}

Crop growth means the growth of cereal crop seedlings, as well as the status and trend of their growth. The upgrowth and development of crop is a complicated process, which is affected by many factors. Many researches showed that the reflective characteristic of crops on red and infra-red has close relation with crop condition, and vegetation index are computed with the reflectivity on the two bands to monitor crop condition, among which the NDVI is mostly used (Zhang Feng, Wu Bingfang, 2004; Xing Suli, 2003). The NDVI is defined as follows (Eq. (1)): 
$\mathrm{NDVI}=(\mathrm{RN}-\mathrm{RR}) /(\mathrm{RN}+\mathrm{RR})$

where RN and RR are atmospherically corrected reflectance at near-infrared and red wavelengths.

Two monitoring methods were used in the system with NDVI data, which are real-time crop growth monitoring and crop growing process monitoring.

\subsection{Real-time crop growth monitoring mothed}

Real-time crop growth monitoring method could get the crop growing status for certain period by comparing the remote sensed data (NDVI, for example) of the period with the data of the period in the history (last year, mostly) (RAO M.V.K, 1982). In this method, the differential result of the two NDVI images is usually classified into several categories which represent different crop conditions. Crop condition map can be made from the classified result, from which the user can see how much and where the crop conditions have either deteriorated, remained unchanged or meliorated.

\subsection{Crop growing process monitoring method}

Crop growth profile monitoring method, which is the contrast between year and year for crop growing profile, can reflect the crop growing continuance during crop growing season (Zhang Feng, Wu Bingfang, 2004). Time series of NDVI image during the crop season are used and crop growth profiles are formed by getting statistical average of NDVI in a certain region such as a province or a country. The crop growth of the year can be evaluated by analyzing the similarity and difference between the crop growing profiles of the year and the year in the history. According to the responding relationship between the curve and the crop condition, diagnostic parameters (eigenvalues) are extracted to estimate the status of crop growth, which realized the quantitative monitoring of crop growth.

Real time monitoring can show the spatial difference of crop condition at a certain period, while crop growing process monitoring can reflect the crop condition through the crop growing season. The system can provide more reliable and more complete monitoring result by combining the two monitoring methods together.

\section{STRUCTURE AND FUNCTION}

Due to its characteristics of multi-missions and huge data processing work, the system was designed in C/S mode which based on LAN. All the data needed by the system are stored and managed on the server side with a Data Base Management System. The data was processed at the client side by reading data from and writing result to the server. The server supports the running of multi-client, which can shorten the data processing time and increase the instantaneity of the monitoring result.

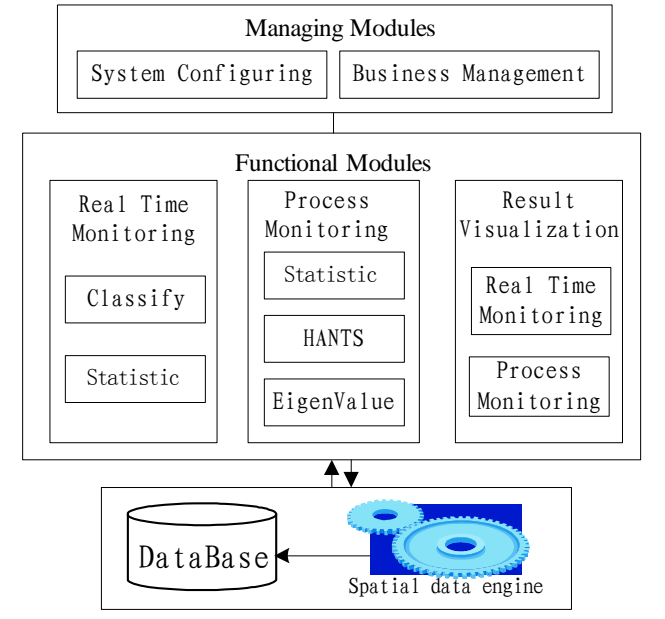

Figure1. System structure

There are five modules in the system on the client side: real time monitoring module, process monitoring module result visualization module, system configuring module and business management module (See Figure 1.). Each module is relatively independent on the other modules and can realize a function by itself while together these modules form an integrated system. The first three modules realized some concrete functions while the rest two modules helped in the run of the system. Each module is described as the following.

\section{(1) Real time monitoring module}

The module realized the real time monitoring method, and was made up of classify and statistic sub-modules. The classify submodule first gets the NDVI difference image of the period and the period in the history, then classify the differential result into 5 categories according to its value. These five categories represent the five-degree of crop conditions that are worse than, little worse than, keep balance to, little better than and better than the period in the history. The thresholds of classification are from expert knowledge. The statistic sub-module can get the percentage of each category of classified result in plowland in a region (such as a country) (figure 2, left). The global land cover data of IGBP (http://edcftp.cr.usgs.gov) was used in the module to distinguish plowland and non-plowland.

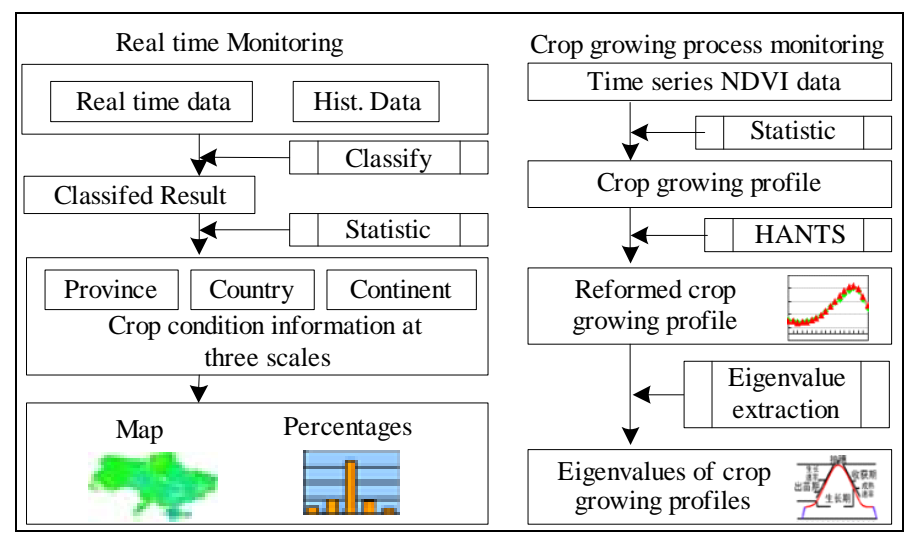

Figure 2. Processing flow of two modules

(2) Process monitoring module 
The module realized the crop growing-process monitoring method and was made up of statistic, HANTS and eigenvalue extraction sub-modules. The statistic sub-module gets the average value of NDVI of plowland in the target region. Time series average value of NDVI for the same region can form the profile of crop growing process. Although the NDVI data used in the system was 16-day (16 day for MODIS, 10 day for NOAA/AVHRR and SOPT/VEGETATION) composite through MVC method, which can alleviate the influence of cloud and other noises, there are still some noises in the crop growing profile. HANTS sub-module can remove these negative influences by re-constructing the profile with HANTS (Harmonic Analysis of Time Series) method (G.J.Roerink, 2000). Then the crop condition can be monitored qualitatively by comparing the crop growing profile of this year and the year in the history (last year usually). Eigenvalues of the profile such as peak value, average, growing rate, etc, are extracted by eigenvalue extraction sub-module, which are used to evaluate the crop condition quantitatively (figure 2, right).

\section{(3) Result visualization module}

The module visualizes the results of the above two monitoring modules and outputs the results as JPG file. The real time monitoring results are displayed in different colors which mean the different degree of crop conditions. The results of crop growing process monitoring are displayed as the contrast of the crop growing profiles of this year and the year in the history. The size and resolution of the output file can be customized by the user from the system interface.

\section{(4) System configuring module}

The module configures system parameters such as file path and data base connecting parameters, etc. These parameters are necessary for the system, so system configuring module is the first module to run after you start the system. Besides, the data base operation such as connecting and disconnecting are also done through this module.

\section{(5) Business management module}

The module shows the business progress of the system, which was based on the coding of the business item of the system. Different symbols are used to express different business statuses, which are work finished, work not finished but can be done now and work not only not finished but also can't be done now (e.g., the data was not propared). The business status is expressed in the form of a 2-dimension table, with date and business as its $\mathrm{X}$ and $\mathrm{Y}$ axes.

Both real-time monitoring and crop growing process monitoring are run at three different scales, which are state (province) scale, country scale and continent scale. Monitoring at country sale was carried out in all the 46 countries and regions while monitoring at province scale was carried out only in countries that have great crop acreage or have close grain trade relation with China, such as United States, India, Australia, Brazil and Russia, etc. Monitoring at province scale can provide much more detailed crop condition information while the monitoring at continent scale supplies the most macroscopical crop condition information. The monitoring target at continent scale includes North America, South America, Africa, Europe and South-east Asia, etc.

\section{SYSTEM IMPLEMENTATION}

The data of the system was managed with Oracle DBMS on the server side, with ArcSDE as the spatial data engine. Client side

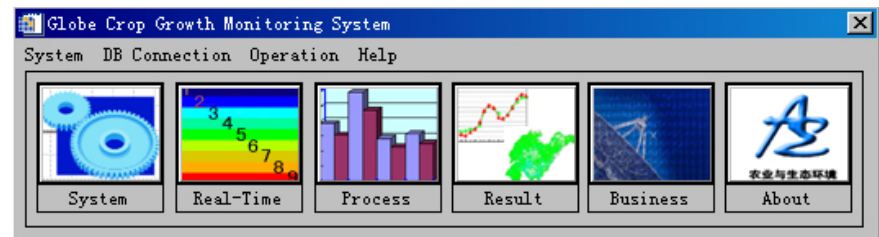

Figure 3. System Interfac

accesses the data base through Oracle client and ODBC.

The system on the client side was developed with the fourthgeneration programming language IDL (Interactive Data Language). The interface of the system is as figure 3 :

The system can be run on the flat of Windows and Linux after implementation. IDL RE (run environment) is needed to support the system.

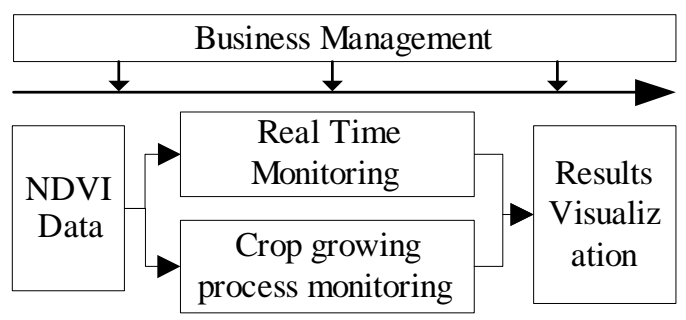

Figure 4. System Runing Flow

\section{RUN OF THE SYSTEM}

The development of the system was finished on 08/2004 and was tested through the rest of the year. In year 2005 the system was put into operation. Its running flow is as figure 4 :

Taken the global NDVI data products downloaded from the net (http://edcimswww.cr.usgs.gov/pub/imswelcome) as data source, the system runs real time monitoring and crop growing process monitoring at the same time. Monitoring results are output to JPG files by results visualization module. The business progress of the system was managed by the business Management module (figure 4).

The system was run successfully in the year 2005 and its results were included in the "China Crop Report with Remote Sensing" (Wu Bingfang, 2004) which is a monthly distributed report to provide decision-supporting information to relevant ministries and commissions. Taken the monitoring results at country scale for example, the monitoring results are as figure 5 and figure 6 .

In real time monitoring, not only the crop condition map but also the percentages of each degree of crop conditions are provided to the user. In the crop growing process monitoring, besides the contrast plot of crop growing profiles, the eigenvalues of the curve are extracted to evaluate the regional crop condition quantitatively. 


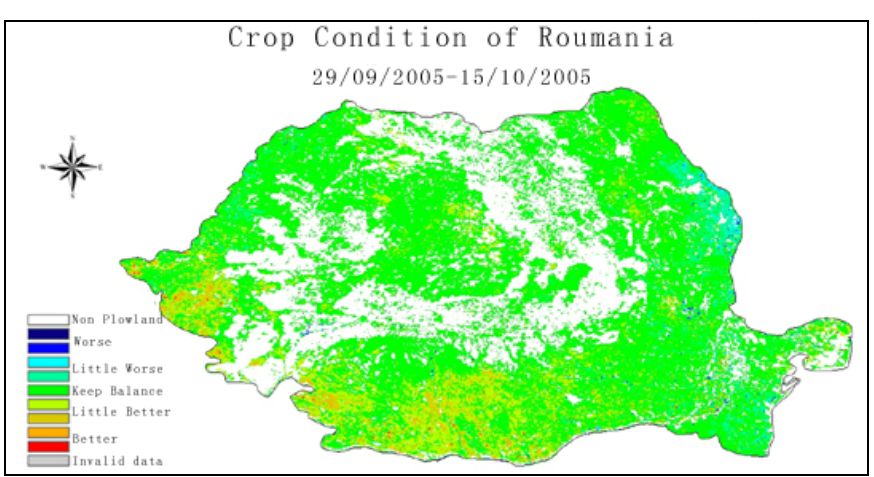

Figure 5. Crop Condition Map of Rumania

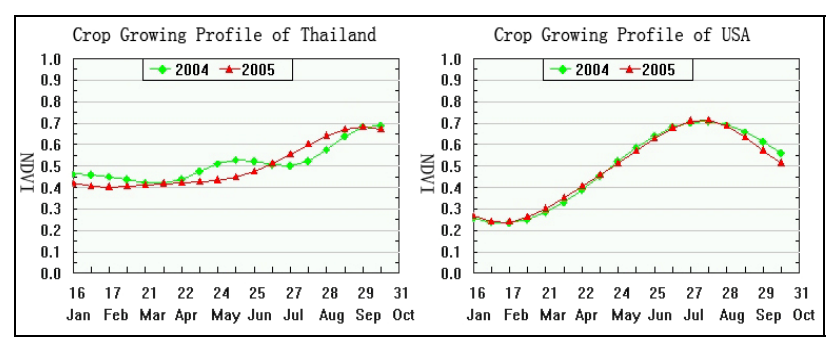

Figure 6. Plots of Crop Growing Profiles

\section{CONCLUSION}

After more than 20 years work, we have accumulated enough experience in method and system construction to build a global crop growth monitoring system. In this condition we built a system that can monitor the global crop growth with remote sensing data. The system realizes the multi-scale global crop growth monitoring by taking the global NDVI products downloaded from the net as data source and using two monitoring method which are real time monitoring and crop growing process monitoring. The system showed the characteristics of fast, effective, high credibility and operational in its run. When the data is prepared, the system needs only 2day's time to finish the data processing of one period which reached the demand to monitor the crop growth instantaneously.

The development of the system was finished in 2004. In 2005, the system was run at monthly frequency to provide crop growth information in 46 countries and regions to relevant departments and ministries of China. The information supplied by the system help them to make correct and scientific decisions.

\section{REFERENCES}

Liu Haiqi, 1999. the intruduction of mars plan of european commission and chinese agriculture remote sensing application.Journal of China Agricultural Resources and Reginal Planning, 20 (3):55-57

Liu Haiqi, 1999. applications of remote sensing in agriculture in the united states. Journal of China Agricultural Resources and Reginal Planning ,20 (2):56 60

M.S.Rassmussen., 1997. Operational yield forests using AVHRR NDVI data: reduction of environmental and inter- annual variablility[J]. International Journal of Remote Sensing. 18(5):1059-1077

Wu Bingfang, 2004. China Crop Watch System with Remote Sensing[J]. Journal of Remote Sensing, 8 (6):482-496

Yang Bangjie, Pei Zhiyuan, Zhou Qinbo, 2002. Key Technologies of Crop Monitoring Using Remote Sensing at a National Scale: Progress and Problems [J]. Transactions of the CSAE, 1 (3):191-195

Xing Suli, Zhang Guanglu, 2003. Application Status quo and prospect of agriculture remote sensing in China [J]. Transactions of the CSAE, 19(6): 174-178

Zhang Feng, Wu Bingfang, Liu Chenlin, 2004. The Integrate Method for Crop growing monitor[J]. Journal of Remote Sensing, 8 (6): 498-514

G.J.Roerink,M.Menenti,, 2000. "Time series of satellite data: development of new products," [C] BCRS-report NRSP-2 99-33, Delft, The Nethelands

RAO M.V.K, Ayyangar R. S, RAO P.P.N., 1982. Role of Multispectral Data in Assessing Crop Management and Crop Yield[C]. Machine Processing of Remote Sensed Data Symposium

Zhang Feng, Wu Bingfang, Liu Chenlin., 2004. A Method For Extract Regional Crop Growth Information with Time Series of NDVI Data [J]. Journal of Remote Sensing, 8 (6): 515-528 